\title{
ON AN INVERSE PROBLEM OF RECONSTRUCTING AN UNKNOWN COEFFICIENT IN A SECOND ORDER HYPERBOLIC EQUATION FROM PARTIAL BOUNDARY MEASUREMENTS*
}

\author{
CHRISTIAN DAVEAU ${ }^{\dagger}$ AND ABDESSATAR KHELIFI $\ddagger$
}

\begin{abstract}
We consider the inverse problem of reconstructing an unknown coefficient in a second order hyperbolic equation from partial (on part of the boundary) dynamic boundary measurements. In this paper we prove that the knowledge of the partial Cauchy data for this class of hyperbolic PDE on any open subset $\Gamma$ of the boundary determines explicitly the coefficient $c$ provided that $c$ is known outside a bounded domain. Then, through construction of appropriate test functions by a geometrical control method, we derive a formula for calculating the coefficient $c$ from the knowledge of the difference between the local Dirichlet to Neumann maps.
\end{abstract}

Key words. Inverse problem, hyperbolic equation, geometric control, identification.

AMS subject classifications. 35R30, 35B40

1. Introduction. In this paper, we present a different method for multidimensional Coefficient Inverse Problems (CIPs) for a class of hyperbolic Partial Differential Equations (PDEs). In the literature, the reader can find many key investigations into this kind of inverse problems, see, e.g. [2, 4, 6, 7, 16, 17, 22, 27, 28, 33] and references cited there. Beilina and Klibanov have deeply studied this important problem in a few recent works $[4,5]$. In [4], the authors have introduced a new globally convergent numerical method to solve a coefficient inverse problem associated with a hyperbolic PDE. The development of globally convergent numerical methods for multidimensional CIPs has started, as a first generation, from the developments found in $[18,19,20]$. Else, Ramm and Rakesh have developed a general method for proving uniqueness theorems for multidimensional inverse problems. For the two dimensional case, Nachman [22] proved an uniqueness result for CIPs for some elliptic equation. Moreover, we find the works of L. Päivärinta and V. Serov [23, 29] about the same issue, but for elliptic equations.

In other manner, the author Chen has treated in [12] the Fourier transform of the hyperbolic equation similar to ours with the unknown coefficient $c(x)$. Unlike this, we derive, using as weights particular background solutions constructed by a geometrical control method, asymptotic formulas in terms of the partial dynamic boundary measurements (Dirichlet to Neumann map) that are caused by the small perturbations. These asymptotic formulae yield the inverse Fourier transform of unknown coefficient.

The ultimate objective of the work described in this paper is to determine, effectively, the unknown smooth coefficient c entering a class of hyperbolic equation in a bounded smooth domain in $\mathbb{R}^{d}$ from partial (on part of the boundary) dynamic boundary measurements. The main difficulty appears in boundary measurements, is that the formulation of our boundary value problem involves unknown boundary values. This problem is well known in the study of the classical elliptic equations, where the characterization of the unknown Neumann boundary value in terms of the

\footnotetext{
*Received August 20, 2010; accepted for publication January 19, 2011.

† Département de Mathématiques, CNRS AGM UMR 8088, Université de Cergy-Pontoise, 95302 Cergy-Pontoise Cedex, France (christian.daveau@math.u-cergy.fr).

‡Département de Mathématiques, Université des Sciences de Carthage, Bizerte, Tunisia (abdessa tar.khelifi@fsb.rnu.tn).
} 
given Dirichlet datum is known as the Dirichlet to Neumann map. But, the problem of determining the unknown boundary values also occurs in the study of hyperbolic equations formulated in a bounded domain.

As our main result we develop, using as weights particular background solutions constructed by a geometrical control method, asymptotic formulas for appropriate averaging of the partial dynamic boundary measurements that are caused by the small perturbations of coefficient according to a parameter $\alpha$. Assume that the coefficient is known outside a bounded domain $\Omega$, and suppose that we know explicitly the value of $\lim _{\alpha \rightarrow 0^{+}} c(x)$ for $x \in \Omega$. Then, the developed asymptotic formulae yield the inverse Fourier transform of the unknown part of this coefficient.

In the subject of small volume perturbations from a known background material associated with the full time-dependent Maxwell's equations, we have derived asymptotic formulas to identify their locations and certain properties of their shapes from dynamic boundary measurements [13]. The present paper represents a different investigation of this line of work.

As closely related stationary identification problems we refer the reader to $[11$, 15, 22,30] and references cited there.

2. Problem formulation. Let $\Omega$ be a bounded, smooth subdomain of $\mathbb{R}^{d}$ with $d \leq 3$, (the assumption $d \leq 3$ is necessary in order to obtain the appropriate regularity for the solution using classical Sobolev embedding, see Brezis [9]). For simplicity we take $\partial \Omega$ to be $\mathcal{C}^{\infty}$, but this condition could be considerably weakened (see Remark 3.1). Let $n=n(x)$ denote the outward unit normal vector to $\Omega$ at a point on $\partial \Omega$. Let $T>0$ be the final time, $x_{0} \in \mathbb{R}^{d} \backslash \bar{\Omega}$ the source position, and let $\Omega^{\prime}$ be a smooth subdomain of $\Omega$. We denote by $\Gamma \subset \subset \partial \Omega$ as a measurable smooth open part of the boundary $\partial \Omega$.

Throughout this paper we shall use quite standard $L^{2}-$ based Sobolev spaces to measure regularity.

As the forward problem, we consider the Cauchy problem for a hyperbolic PDE

$$
\begin{aligned}
c(x) v_{t t}-\Delta v & =0 \quad \text { in } \mathbb{R}^{d} \times(0, T) \\
v(x, 0)=0, v_{t}(x, 0) & =\delta\left(x-x_{0}\right)+\chi(\Omega) \psi,
\end{aligned}
$$

where $\chi(\Omega)$ is the characteristic function of $\Omega$ and $\psi \in \mathcal{C}^{\infty}\left(\mathbb{R}^{d}\right)$ that $\psi(x) \neq 0, \forall x \in \bar{\Omega}$.

Equation (1) governs a wide range of applications, including e.g., propagation of acoustic and electromagnetic waves.

We assume that the coefficient $c(x)$ of equation (1) is such that

$$
c(x)= \begin{cases}c_{\alpha}(x)=c_{0}(x)+\alpha c_{1}(x) & \text { for } x \in \Omega, \\ c_{2}(x)=\text { const } .>0 & \text { for } x \in \mathbb{R}^{d} \backslash \Omega ;\end{cases}
$$

where $c_{i}(x) \in \mathcal{C}^{2}(\bar{\Omega})$ for $i=0,1$ with

$$
c_{1} \equiv 0 \text { in } \Omega \backslash \overline{\Omega^{\prime}}, \quad \text { and } M:=\sup \left\{c_{1}(x) ; x \in \Omega^{\prime}\right\},
$$

where $\Omega^{\prime}$ is a smooth subdomain of $\Omega$ and $M$ is a positive constant. We also assume that $\alpha>0$, the order of magnitude of the small perturbations of coefficient, is sufficiently small that

$$
\left|c_{\alpha}(x)\right| \geq c_{*}>0, x \in \bar{\Omega}
$$


where $c_{*}$ is a positive constant.

REMARK 2.1. Assumptions (3)-(4)-(5) will be useful in section 3. Moreover, assumption (3) implies that the knowledge of the coefficient $c_{2}$ allows us to recover the coefficient $c$ only in the bounded domain $\Omega$.

Now by using (1)-(2), we can introduce the following initial boundary value problem in the domain $\Omega \times(0, T)$ :

$$
\left\{\begin{array}{l}
\left(c_{\alpha} \partial_{t}^{2}-\Delta\right) u_{\alpha}=0 \quad \text { in } \Omega \times(0, T), \\
\left.u_{\alpha}\right|_{t=0}=\varphi,\left.\partial_{t} u_{\alpha}\right|_{t=0}=\psi \quad \text { in } \Omega, \\
\left.u_{\alpha}\right|_{\partial \Omega \times(0, T)}=f
\end{array}\right.
$$

Define $u$ to be the solution of the hyperbolic equation in the homogeneous situation $(\alpha=0)$. Thus, $u$ satisfies

$$
\left\{\begin{array}{l}
\left(c_{0} \partial_{t}^{2}-\Delta\right) u=0 \quad \text { in } \Omega \times(0, T), \\
\left.u\right|_{t=0}=\varphi,\left.\partial_{t} u\right|_{t=0}=\psi \quad \text { in } \Omega, \\
\left.u\right|_{\partial \Omega \times(0, T)}=f .
\end{array}\right.
$$

Here $\varphi \in \mathcal{C}^{\infty}(\bar{\Omega})$ and $f \in \mathcal{C}^{\infty}\left(0, T ; \mathcal{C}^{\infty}(\partial \Omega)\right)$ are subject to the compatibility conditions

$$
\left.\partial_{t}^{2 l} f\right|_{t=0}=\left.\left(\Delta^{l} \varphi\right)\right|_{\partial \Omega} \text { and }\left.\partial_{t}^{2 l+1} f\right|_{t=0}=\left.\left(\Delta^{l} \psi\right)\right|_{\partial \Omega}, \quad l=1,2, \ldots
$$

which give that (7) has a unique solution in $\mathcal{C}^{\infty}([0, T] \times \bar{\Omega})$, see [14]. It is also wellknown that (6) has a unique weak solution $u_{\alpha} \in \mathcal{C}^{0}\left(0, T ; H^{1}(\Omega)\right) \cap \mathcal{C}^{1}\left(0, T ; L^{2}(\Omega)\right)$, see [21], [14]. Indeed, from [21] we have that $\left.\frac{\partial u_{\alpha}}{\partial n}\right|_{\partial \Omega}$ belongs to $L^{2}\left(0, T ; L^{2}(\partial \Omega)\right)$.

Now, we define $\Gamma_{c}:=\partial \Omega \backslash \bar{\Gamma}$, and the trace space

$$
\widetilde{H}^{\frac{1}{2}}(\Gamma)=\left\{v \in H^{\frac{1}{2}}(\partial \Omega \times(0, T)), v \equiv 0 \text { on } \Gamma_{c} \times(0, T)\right\} .
$$

It is known that the dual of $\widetilde{H}^{\frac{1}{2}}(\Gamma)$ is $H^{-\frac{1}{2}}(\Gamma)$.

Moreover, we introduce the function $\tilde{f}=\chi(\Gamma) f$ for $(x, t) \in \partial \Omega \times(0, T)$, where $\chi(\Gamma)$ is the characteristic function of $\Gamma$. Then, one can write

$$
\tilde{f}=\left.f\right|_{\Gamma \times(0, T)} \in \widetilde{H}^{\frac{1}{2}}(\Gamma) .
$$

Therefore, we can define the local Dirichlet to Neumann map (D-t-N) operator associated with coefficient $c_{\alpha}$ by :

$$
\Lambda_{\alpha}(\tilde{f})=\left.\frac{\partial u_{\alpha}}{\partial n}\right|_{\Gamma} \text { for } \tilde{f} \in \widetilde{H}^{\frac{1}{2}}(\Gamma),
$$

where $u_{\alpha}$ solves the problem (6). Let $u$ denote the solution of the hyperbolic equation (7) with the Dirichlet boundary condition $u=f$ on $\partial \Omega \times(0, T)$. Then, the local Dirichlet to Neumann map associated with $c_{0}$ is $\Lambda_{0}(\tilde{f})=\left.\frac{\partial u}{\partial n}\right|_{\Gamma}$ for $\tilde{f} \in \widetilde{H}^{\frac{1}{2}}(\Gamma)$. 
The proposed inverse problem can be formulated as a problem of finding the coefficient $c(x)$ from $\left(\Lambda_{\alpha}-\Lambda_{0}\right)(\tilde{f})$ as follows.

InVERSE Problem 2.1. Let $\Omega$ be a bounded, smooth subdomain of $\mathbb{R}^{d}$ with $d \leq 3$. Given the function $\tilde{f}$ defined by (8) where the boundary data $f$ was given in (6)-(7). Assume that the coefficient $c_{1}(x)$ is unknown in the domain $\Omega$, the two other coefficients $c_{0}(x), c_{2}(x)$ are well known. Find the coefficient $c_{\alpha}(x)=c_{0}(x)+\alpha c_{1}(x)$ from the knowledge of the difference between the local Dirichlet to Neumann maps $\left(\Lambda_{\alpha}-\Lambda_{0}\right)(\tilde{f})$ on $\Gamma$.

We may illustrate the justification of the proposed Inverse Problem 2.1 as follows.

REMARK 2.2. The term $\alpha c_{2}(x)$ in (3) can be regarded as small amplitude perturbations of the background coefficient $c_{0}$ for the smooth coefficient $c(x)$. Our procedure may be expected to lead to computational identification algorithms, aimed at determining electromagnetic parameters (for example) of an object based on partial dynamic boundary measurements. Moreover, in our proposed inverse problem, we suggest that the method is quite stable with respect to noise in measurements and errors in the different approximations.

In practice, we will develop an asymptotic expansions of an "appropriate averaging" of $\frac{\partial u_{\alpha}}{\partial n}$ on $\Gamma \times(0, T)$, using particular background solutions as weights. These particular solutions are constructed by a control method as it has been done in the original work [33] (see also [8], [10], [24] and [25]). It has been known for some time that the full knowledge of the (hyperbolic) Dirichlet to Neumann map $\left(\left.\left.u_{\alpha}\right|_{\partial \Omega \times(0, T)} \mapsto \frac{\partial u_{\alpha}}{\partial n}\right|_{\partial \Omega \times(0, T)}\right)$ uniquely determines conductivity, see [26], [31]. Our identification procedure can be regarded as an important attempt to generalize the results of [26] and [31] in the case of partial knowledge (i.e., on only part of the boundary) of the Dirichlet to Neumann map to determine the coefficient of the hyperbolic equation considered above. The question of uniqueness of this inverse problem can be addressed positively via the method of Carleman estimates, see, e.g., $[17,19]$.

3. The identification procedure. Before describing our identification procedure, let us introduce the following cutoff function $\beta(x) \in \mathcal{C}_{0}^{\infty}(\Omega)$ such that $\beta \equiv 1$ on $\Omega^{\prime}$ and let $\eta \in \mathbb{R}^{d}$.

We will take in what follows $\varphi(x)=e^{i \eta \cdot x}, \psi(x)=-i|\eta| e^{i \eta \cdot x}$, and $f(x, t)=e^{i \eta \cdot x-i|\eta| t}$ and assume that we are in possession of the boundary measurements of

$$
\frac{\partial u_{\alpha}}{\partial n} \quad \text { on } \Gamma \times(0, T)
$$

This particular choice of data $\varphi, \psi$, and $f$ implies that the background solution $u$ of the wave equation (7) in the homogeneous background medium can be given explicitly.

Suppose now that $T$ and the part $\Gamma$ of the boundary $\partial \Omega$ are such that they geometrically control $\Omega$ which roughly means that every geometrical optic ray, starting at any point $x \in \Omega$ at time $t=0$ hits $\Gamma$ before time $T$ at a non diffractive point, see [3]. It follows from [32] (see also [1]) that there exists (a unique) $g_{\eta} \in H_{0}^{1}\left(0, T ; T L^{2}(\Gamma)\right.$ ) 
(constructed by the Hilbert Uniqueness Method) such that the unique weak solution $w_{\eta}$ to the wave equation

$$
\left\{\begin{array}{l}
\left(c_{0} \partial_{t}^{2}-\Delta\right) w_{\eta}=0 \quad \text { in } \Omega \times(0, T) \\
\left.w_{\eta}\right|_{t=0}=\beta(x) e^{i \eta \cdot x} \in H_{0}^{1}(\Omega) \\
\left.\partial_{t} w_{\eta}\right|_{t=0}=0 \quad \text { in } \Omega \\
\left.w_{\eta}\right|_{\Gamma \times(0, T)}=g_{\eta} \\
\left.w_{\eta}\right|_{\partial \Omega \backslash \bar{\Gamma} \times(0, T)}=0
\end{array}\right.
$$

satisfies $w_{\eta}(T)=\partial_{t} w_{\eta}(T)=0$.

Let $\theta_{\eta} \in H^{1}\left(0, T ; L^{2}(\Gamma)\right)$ denote the unique solution of the Volterra equation of second kind

$$
\left\{\begin{array}{l}
\partial_{t} \theta_{\eta}(x, t)+\int_{t}^{T} e^{-i|\eta|(s-t)}\left(\theta_{\eta}(x, s)-i|\eta| \partial_{t} \theta_{\eta}(x, s)\right) d s \\
\theta_{\eta}(x, 0)=0 \quad \text { for } x \in \Gamma .
\end{array}\right.
$$

We can refer to the work of Yamamoto in [33] who conceived the idea of using such Volterra equation to apply the geometrical control for solving inverse source problems.

The existence and uniqueness of this $\theta_{\eta}$ in $H^{1}\left(0, T ; L^{2}(\Gamma)\right)$ for any $\eta \in \mathbb{R}^{d}$ can be established using the resolvent kernel. However, observing from differentiation of (10) with respect to $t$ that $\theta_{\eta}$ is the unique solution of the ODE:

$$
\left\{\begin{array}{l}
\partial_{t}^{2} \theta_{\eta}-\theta_{\eta}=e^{i|\eta| t} \partial_{t}\left(e^{-i|\eta| t} g_{\eta}\right) \quad \text { for } x \in \Gamma, t \in(0, T) \\
\theta_{\eta}(x, 0)=0, \partial_{t} \theta_{\eta}(x, T)=0 \quad \text { for } x \in \Gamma
\end{array}\right.
$$

the function $\theta_{\eta}$ may be find (in practice) explicitly with variation of parameters and it also immediately follows from this observation that $\theta_{\eta}$ belongs to $H^{2}\left(0, T ; L^{2}(\Gamma)\right)$. We introduce $v_{\eta}$ as the unique weak solution (obtained by transposition) in $\mathcal{C}^{0}\left(0, T ; L^{2}(\Omega)\right) \cap \mathcal{C}^{1}\left(0, T ; H^{-1}(\Omega)\right)$ to the wave equation

$$
\left\{\begin{array}{l}
\left(c_{0} \partial_{t}^{2}-\Delta\right) v_{\eta}=0 \quad \text { in } \Omega \times(0, T), \\
\left.v_{\eta}\right|_{t=0}=0 \quad \text { in } \Omega \\
\left.\partial_{t} v_{\alpha, \eta}\right|_{t=0}=i \nabla \cdot\left(\eta c_{1}(x) e^{i \eta \cdot x}\right) \in L^{2}(\Omega), \\
\left.v_{\eta}\right|_{\partial \Omega \times(0, T)}=0 .
\end{array}\right.
$$

Then, the following holds.

Proposition 3.1. Suppose that $\Gamma$ and $T$ geometrically control $\Omega$. For any $\eta \in \mathbb{R}^{d}$ we have

$$
\int_{0}^{T} \int_{\Gamma} g_{\eta} \Lambda_{0}\left(v_{\eta}\right) d \sigma(x) d t=|\eta|^{2} \int_{\Omega^{\prime}} c_{1}(x) e^{2 i \eta \cdot x} d x
$$

Here $d \sigma(x)$ means an elementary surface for $x \in \Gamma$. 
Proof. Let $v_{\eta}$ be the solution of (12). From [21] [Theorem 4.1, page 44] it follows that $\Lambda_{0}\left(v_{\eta}\right)=\left.\frac{\partial v_{\eta}}{\partial n}\right|_{\Gamma} \in L^{2}\left(0, T ; L^{2}(\Gamma)\right)$. Then, multiply the equation $\left(\partial_{t}^{2}+\Delta\right) v_{\eta}=0$ by $w_{\eta}$ and integrating by parts over $(0, T) \times \Omega$, for any $\eta \in \mathbb{R}^{d}$ we have

$$
\int_{0}^{T} \int_{\Omega}\left(\partial_{t}^{2}-\Delta\right) v_{\eta} w_{\eta}=i \int_{\Omega} \nabla \cdot\left(\eta c_{1}(x) e^{i \eta \cdot x}\right) \beta(x) e^{i \eta \cdot x} d x-\int_{0}^{T} \int_{\Gamma} g_{\eta} \frac{\partial v_{\eta}}{\partial n}=0 .
$$

Therefore

$$
|\eta|^{2} \int_{\Omega^{\prime}} c_{1}(x) e^{2 i \eta \cdot x} d x=\int_{0}^{T} \int_{\Gamma} g_{\eta} \frac{\partial v_{\eta}}{\partial n}
$$

since $c_{1} \equiv 0$ on $\Omega \backslash \overline{\Omega^{\prime}}$.

In term of the function $v_{\eta}$ as solution of (10), we introduce

$$
\tilde{u}_{\alpha}(x, t)=u(x, t)+\alpha^{d} \int_{0}^{t} e^{-i|\eta| s} v_{\eta}(x, t-s) d s, x \in \Omega, t \in(0, T) .
$$

Moreover, for $z(t) \in \mathcal{C}_{0}^{\infty}(] 0, T[)$ and for any $v \in L^{1}\left(0, T ; L^{2}(\Omega)\right)$, we define

$$
\hat{v}(x)=\int_{0}^{T} v(x, t) z(t) d t \in L^{2}(\Omega) .
$$

The following lemma is useful to proof our main result.

LEMMA 3.1. Consider an arbitrary function $c(x)$ satisfying condition (3) and assume that conditions (4) and (5) hold. Let $u, u_{\alpha}$ be solutions of (7) and (6) respectively. Then, using (15) the following estimates hold:

$$
\left\|u_{\alpha}-u\right\|_{L^{\infty}\left(0, T ; L^{2}(\Omega)\right)} \leq C \alpha
$$

where $C$ a positive constant. And,

$$
\left\|\tilde{u}_{\alpha}-u_{\alpha}\right\|_{L^{\infty}\left(0, T ; L^{2}(\Omega)\right)} \leq C^{\prime} \alpha^{d+1},
$$

where $C^{\prime}$ is a positive constant.

Proof. Let $y_{\alpha}$ be defined by

$$
\left\{\begin{array}{l}
y_{\alpha} \in H_{0}^{1}(\Omega) \\
\Delta y_{\alpha}=c_{\alpha} \partial_{t}\left(u_{\alpha}-u\right) \quad \text { in } \Omega
\end{array}\right.
$$

We have

$$
\int_{\Omega} c_{\alpha} \partial_{t}^{2}\left(u_{\alpha}-u\right) y_{\alpha}+\int_{\Omega} \nabla\left(u_{\alpha}-u\right) \cdot \nabla y_{\alpha}=\alpha \int_{\Omega} \frac{c_{1}}{c_{0}} \nabla u \cdot \nabla y_{\alpha}
$$

Since

$$
\int_{\Omega} \nabla\left(u_{\alpha}-u\right) \cdot \nabla y_{\alpha}=-\int_{\Omega} c_{\alpha} \partial_{t}\left(u_{\alpha}-u\right)\left(u_{\alpha}-u\right)=-\frac{1}{2} \partial_{t} \int_{\Omega} c_{\alpha}\left(u_{\alpha}-u\right)^{2},
$$

and

$$
\int_{\Omega} c_{\alpha} \partial_{t}^{2}\left(u_{\alpha}-u\right) y_{\alpha}=-\frac{1}{2} \partial_{t} \int_{\Omega}\left|\nabla y_{\alpha}\right|^{2}
$$


we obtain by using (4) that

$$
\partial_{t} \int_{\Omega}\left|\nabla y_{\alpha}\right|^{2}+\partial_{t} \int_{\Omega} c_{\alpha}\left(u_{\alpha}-u\right)^{2}=-2 \alpha \int_{\Omega} \frac{c_{1}}{c_{0}} \nabla u \cdot \nabla y_{\alpha} \leq C \alpha\left\|\nabla y_{\alpha}\right\|_{L^{\infty}\left(0, T ; L^{2}(\Omega)\right)},
$$

where $C=C\left(M, \Omega^{\prime}\right)$.

From the Gronwall Lemma it follows that

$$
\left\|u_{\alpha}-u\right\|_{L^{\infty}\left(0, T ; L^{2}(\Omega)\right)} \leq C \alpha .
$$

As a consequence, by using (16) one can see that the function $\hat{u}_{\alpha}-\hat{u}$ solves the following boundary value problem

$$
\left\{\begin{array}{l}
\Delta\left(\hat{u}_{\alpha}-\hat{u}\right)=O(\alpha) \text { in } \Omega \\
\left.\left(\hat{u}_{\alpha}-\hat{u}\right)\right|_{\partial \Omega}=0
\end{array}\right.
$$

Integration by parts immediately gives,

$$
\left\|\operatorname{grad}\left(\hat{u}_{\alpha}-\hat{u}\right)\right\|_{L^{2}(\Omega)}=O(\alpha) .
$$

Taking into account that $\operatorname{grad}\left(u_{\alpha}-u\right) \in L^{\infty}\left(0, T ; L^{2}(\Omega)\right)$, we find by using the above estimate that

$$
\left\|\operatorname{grad}\left(u_{\alpha}-u\right)\right\|_{L^{2}(\Omega)}=O(\alpha) \text { a.e. } t \in(0, T) .
$$

Under relation (15), one can define the function $\tilde{y}_{\alpha}$ as solution of

$$
\left\{\begin{array}{l}
\tilde{y}_{\alpha} \in H_{0}^{1}(\Omega) \\
\Delta \tilde{y}_{\alpha}=c_{\alpha} \partial_{t}\left(\tilde{u}_{\alpha}-u_{\alpha}\right) \text { in } \Omega .
\end{array}\right.
$$

Integrating by parts immediately yields

$$
\int_{\Omega} c_{\alpha} \partial_{t}^{2}\left(\tilde{u}_{\alpha}-u_{\alpha}\right) \tilde{y}_{\alpha}=-1 / 2 \partial_{t} \int_{\Omega}\left|\nabla \tilde{y}_{\alpha}\right|^{2}
$$

and

$$
\int_{\Omega} \nabla\left(\tilde{u}_{\alpha}-u_{\alpha}\right) \nabla \tilde{y}_{\alpha}=-1 / 2 \partial_{t} \int_{\Omega} c_{\alpha}\left(\tilde{u}_{\alpha}-u_{\alpha}\right)^{2}
$$

To proceed with the proof of estimate (17), we firstly remark that the function $\tilde{u}_{\alpha}$ given by (15) is a solution of

$$
\left\{\begin{array}{l}
\left(c_{0} \partial_{t}^{2}-\Delta\right) \tilde{u}_{\alpha}=i \alpha^{d} \nabla \cdot\left(\eta c_{1}(x) e^{i \eta \cdot x}\right) e^{-i|\eta| t} \in L^{2}(\Omega) \quad \text { in } \Omega \times(0, T), \\
\left.\tilde{u}_{\alpha}\right|_{t=0}=\varphi(x) \quad \text { in } \Omega \\
\left.\partial_{t} \tilde{u}_{\alpha}\right|_{t=0}=\psi(x) \quad \text { in } \Omega \\
\left.\tilde{u}_{\alpha}\right|_{\partial \Omega \times(0, T)}=e^{i \eta \cdot x-i|\eta| t} .
\end{array}\right.
$$

Then, we deduce that $u_{\alpha}-\tilde{u}_{\alpha}$ solves the following initial boundary value problem,

$$
\left\{\begin{array}{l}
\left(c_{\alpha} \partial_{t}^{2}-\nabla \cdot \Delta\right)\left(u_{\alpha}-\tilde{u}_{\alpha}\right) \\
=\alpha^{d} \nabla \cdot\left(c_{1}(x) \operatorname{grad}\left(\int_{0}^{t} e^{-i|\eta| s} v_{\eta}(x, t-s) d s\right)\right) \quad \text { in } \Omega \times(0, T), \\
\left.\left(u_{\alpha}-\tilde{u}_{\alpha}\right)\right|_{t=0}=0 \quad \text { in } \Omega \\
\left.\partial_{t}\left(u_{\alpha}-\tilde{u}_{\alpha}\right)\right|_{t=0}=0 \quad \text { in } \Omega \\
\left.\left(u_{\alpha}-\tilde{u}_{\alpha}\right)\right|_{\partial \Omega \times(0, T)}=0
\end{array}\right.
$$


Finally, we can use (21) to find by integrating by parts that

$$
\partial_{t} \int_{\Omega}\left|\nabla \tilde{y}_{\alpha}\right|^{2}+\partial_{t} \int_{\Omega} c_{\alpha}\left(\tilde{u}_{\alpha}-u_{\alpha}\right)^{2}=2 \alpha^{d} \int_{\Omega} c_{1} \operatorname{grad}\left(u-u_{\alpha}\right) \cdot \operatorname{grad} \tilde{y}_{\alpha}
$$

which, from the Gronwall Lemma and by using both relations (5), (20), yields

$$
\left\|\tilde{u}_{\alpha}-u_{\alpha}\right\|_{L^{\infty}\left(0, T ; L^{2}(\Omega)\right)} \leq C^{\prime} \alpha^{d+1} .
$$

This achieves the proof.

Now, we identify the function $c(x)$ by using the difference between local Dirichlet to Neumann maps and the function $\theta_{\eta}$ as solution to the Volterra equation (10) or equivalently the $\operatorname{ODE}(11)$, as a function of $\eta$. Then, the following main result holds.

TheOREM 3.1. Let $\eta \in \mathbb{R}^{d}, d=2,3$. Suppose that the smooth coefficient $c(x)$ satisfies (3)-(4)-(5). Let $u_{\alpha}$ be the unique solution in $\mathcal{C}^{0}\left(0, T ; H^{1}(\Omega)\right) \cap \mathcal{C}^{1}\left(0, T ; L^{2}(\Omega)\right)$ to the wave equation (6) with $\varphi(x)=e^{i \eta \cdot x}, \psi(x)=-i|\eta| e^{i \eta \cdot x}$, and $f(x, t)=e^{i \eta \cdot x-i|\eta| t}$. Let $\tilde{f}$ be the function which satisfies (8). Suppose that $\Gamma$ and $T$ geometrically control $\Omega$, then we have

$$
\begin{aligned}
& \int_{0}^{T} \int_{\Gamma}\left(\theta_{\eta}+\partial_{t} \theta_{\eta} \partial_{t} \cdot\right)\left(\Lambda_{\alpha}-\Lambda_{0}\right)(\tilde{f})(x, t) d \sigma(x) d t \\
= & \alpha^{d-1}|\eta|^{2} \int_{\Omega^{\prime}}\left(c_{\alpha}-c_{0}\right)(x) e^{2 i \eta \cdot x} d x+O\left(\alpha^{d+1}\right) \\
= & \alpha^{d}|\eta|^{2} \int_{\Omega^{\prime}} c_{1}(x) e^{2 i \eta \cdot x} d x+O\left(\alpha^{d+1}\right),
\end{aligned}
$$

where $\theta_{\eta}$ is the unique solution to the ODE (11) with $g_{\eta}$ defined as the boundary control in (9). The term $O\left(\alpha^{d+1}\right)$ is independent of the function $c_{1}$. It depends only on the bound $M$.

Proof. Since the extension of $\left(\Lambda_{\alpha}-\Lambda_{0}\right)(\tilde{f})(x, t)$ to $\partial \Omega \times(0, T)$ is $\left(\frac{\partial u_{\alpha}}{\partial n}-\frac{\partial u}{\partial n}\right)$, then by conditions $\partial_{t} \theta_{\eta}(T)=0$ and $\left.\left(\frac{\partial u_{\alpha}}{\partial n}-\frac{\partial u}{\partial n}\right)\right|_{t=0}=0$, we have $\left.\left(\Lambda_{\alpha}-\Lambda_{0}\right)(\tilde{f})(x, t)\right|_{t=0}=0$. Therefore the term

$$
\int_{0}^{T} \int_{\Gamma} \partial_{t} \theta_{\eta} \partial_{t}\left(\Lambda_{\alpha}-\Lambda_{0}\right)(\tilde{f})(x, t) d \sigma(x) d t
$$

may be simplified as follows

$$
\begin{aligned}
& \int_{0}^{T} \int_{\Gamma} \partial_{t} \theta_{\eta} \partial_{t}\left(\Lambda_{\alpha}-\Lambda_{0}\right)(\tilde{f})(x, t) d \sigma(x) d t \\
= & -\int_{0}^{T} \int_{\Gamma} \partial_{t}^{2} \theta_{\eta}\left(\Lambda_{\alpha}-\Lambda_{0}\right)(\tilde{f})(x, t) d \sigma(x) d t .
\end{aligned}
$$


On the other hand, we have

$$
\begin{aligned}
& \int_{0}^{T} \int_{\Gamma}\left[\theta_{\eta}\left(\Lambda_{\alpha}-\Lambda_{0}\right)(\tilde{f})+\partial_{t} \theta_{\eta} \partial_{t}\left(\Lambda_{\alpha}-\Lambda_{0}\right)(\tilde{f})\right](x, t) d \sigma(x) d t \\
& =\int_{0}^{T} \int_{\Gamma}\left[\theta_{\eta}\left(\Lambda_{\alpha}(\tilde{f})-\tilde{\Lambda}_{\alpha}\left(\left.\tilde{u}_{\alpha}\right|_{\Gamma \times(0, T)}\right)\right)\right. \\
& +\partial_{t} \theta_{\eta} \partial_{t}\left(\Lambda_{\alpha}(\tilde{f})-\tilde{\Lambda}_{\alpha}\left(\left.\tilde{u}_{\alpha}\right|_{\Gamma \times(0, T)}\right)\right](x, t) d \sigma(x) d t \\
& +\int_{0}^{T} \int_{\Gamma}\left[\theta_{\eta} \alpha^{d} \int_{0}^{t} e^{-i|\eta| s} \frac{\partial v_{\eta}}{\partial n}(x, t-s) d s\right. \\
& \left.+\alpha^{d} \partial_{t} \theta_{\eta} \partial_{t} \int_{0}^{t} e^{-i|\eta| s} \frac{\partial v_{\eta}}{\partial n}(x, t-s) d s\right] d \sigma(x) d t
\end{aligned}
$$

where $\tilde{\Lambda}_{\alpha}\left(\left.\tilde{u}_{\alpha}\right|_{\Gamma \times(0, T)}\right)=\Lambda_{0}(\tilde{f})+\alpha^{d} \int_{0}^{t} e^{-i|\eta| s} \Lambda_{0}\left(\left.v_{\eta}\right|_{\Gamma}\right)(x, t-s) d s$.

Given that, $\theta_{\eta}$ satisfies the Volterra equation (11) and

$$
\begin{aligned}
& \partial_{t}\left(\int_{0}^{t} e^{-i|\eta| s} \frac{\partial v_{\eta}}{\partial n}(x, t-s) d s\right)=\partial_{t}\left(-e^{-i|\eta| t} \int_{0}^{t} e^{i|\eta| s} \frac{\partial v_{\eta}}{\partial n}(x, s) d s\right) \\
& =i|\eta| e^{-i|\eta| t} \int_{0}^{t} e^{i|\eta| s} \frac{\partial v_{\eta}}{\partial n}(x, s) d s+\frac{\partial v_{\eta}}{\partial n}(x, t)
\end{aligned}
$$

we obtain by integrating by parts over $(0, T)$ that

$$
\begin{aligned}
\int_{0}^{T} \int_{\Gamma}\left[\theta_{\eta} \int_{0}^{t} e^{-i|\eta| s} \frac{\partial v_{\eta}}{\partial n}(x, t-s) d s+\partial_{t} \theta_{\eta} \partial_{t} \int_{0}^{t} e^{-i|\eta| s} \frac{\partial v_{\eta}}{\partial n}(x, t-s) d s\right] d \sigma(x) d t \\
=\int_{0}^{T} \int_{\Gamma}\left(\frac{\partial v_{\eta}}{\partial n}(x, t)\left(\partial_{t} \theta_{\eta}+\int_{t}^{T} \theta_{\eta}(s) e^{i|\eta|(t-s)} d s\right)\right. \\
\left.\quad-i|\eta|\left(e^{-i|\eta| t} \partial_{t} \theta_{\eta}(t)\right) \int_{0}^{t} e^{i|\eta| s} \frac{\partial v_{\eta}}{\partial n}(x, s) d s\right) d \sigma(x) d t \\
=\int_{0}^{T} \int_{\Gamma} \frac{\partial v_{\eta}}{\partial n}(x, t)\left(\partial_{t} \theta_{\eta}+\int_{t}^{T}\left(\theta_{\eta}(s)-i|\eta| \partial_{t} \theta_{\eta}(s)\right) e^{i|\eta|(t-s)} d s\right) d \sigma(x) d t \\
=\int_{0}^{T} \int_{\Gamma} g_{\eta}(x, t) \Lambda_{0}\left(\left.v_{\eta}\right|_{\Gamma}\right)(x, t) d \sigma(x) d t
\end{aligned}
$$

and so, from Proposition 3.1 we obtain

$$
\begin{aligned}
& \int_{0}^{T} \int_{\Gamma}\left[\theta_{\eta}\left(\Lambda_{\alpha}-\Lambda_{0}\right)(\tilde{f})+\partial_{t} \theta_{\eta} \partial_{t}\left(\Lambda_{\alpha}-\Lambda_{0}\right)(\tilde{f})\right](x, t) d \sigma(x) d t \\
& =\alpha^{d}|\eta|^{2} \int_{\Omega^{\prime}} c_{1}(x) e^{2 i \eta \cdot x} d x+\int_{0}^{T} \int_{\Gamma}\left[\theta_{\eta}\left(\Lambda_{\alpha}(\tilde{f})-\tilde{\Lambda}_{\alpha}\left(\left.\tilde{u}_{\alpha}\right|_{\Gamma \times(0, T)}\right)\right)\right. \\
& \left.\quad+\partial_{t} \theta_{\eta} \partial_{t}\left(\Lambda_{\alpha}(\tilde{f})-\tilde{\Lambda}_{\alpha}\left(\left.\tilde{u}_{\alpha}\right|_{\Gamma \times(0, T)}\right)\right)\right] d \sigma(x) d t+O\left(\alpha^{d+1}\right) .
\end{aligned}
$$

Thus, to prove Theorem 3.1 it suffices then to show that $\int_{0}^{T} \int_{\Gamma}\left[\theta_{\eta}\left(\Lambda_{\alpha}(\tilde{f})-\tilde{\Lambda}_{\alpha}\left(\left.\tilde{u}_{\alpha}\right|_{\Gamma \times(0, T)}\right)\right)+\partial_{t} \theta_{\eta} \partial_{t}\left(\Lambda_{\alpha}(\tilde{f})-\tilde{\Lambda}_{\alpha}\left(\left.\tilde{u}_{\alpha}\right|_{\Gamma \times(0, T)}\right)\right)\right] d \sigma(x) d t=O\left(\alpha^{d+1}\right)$. 
From definition (16) we have

$$
\hat{u}_{\alpha}-\hat{\tilde{u}}_{\alpha}=\int_{0}^{T}\left(u_{\alpha}-\tilde{u}_{\alpha}\right) z(t) d t
$$

which gives by system $(21)$ that

$$
\begin{aligned}
& \Delta\left(\hat{u}_{\alpha}-\hat{\tilde{u}}_{\alpha}\right) \\
& =\int_{0}^{T} c_{\alpha} \partial_{t}^{2}\left(u_{\alpha}-\tilde{u}_{\alpha}\right) z(t) d t+\alpha^{d} \int_{0}^{T} \nabla \cdot\left(c_{1}(x) \operatorname{grad}\left(\int_{0}^{t} e^{-i|\eta| s} v_{\eta}(x, t-s) d s\right)\right) z(t) d t .
\end{aligned}
$$

Thus, by (15) and (21) again, we see that the function $\hat{u}_{\alpha}-\hat{\tilde{u}}_{\alpha}$ is solution of

$$
\left\{\begin{array}{l}
-\Delta\left(\hat{u}_{\alpha}-\hat{\tilde{u}}_{\alpha}\right)=-\int_{0}^{T} c_{\alpha}\left(u_{\alpha}-\tilde{u}_{\alpha}\right) z^{\prime \prime}(t) d t+\nabla \cdot\left(c_{1}(x) \operatorname{grad}\left(\hat{\tilde{u}}_{\alpha}-\hat{u}\right)\right) \text { in } \Omega \\
\left.\left(\hat{u}_{\alpha}-\hat{\tilde{u}}_{\alpha}\right)\right|_{\partial \Omega}=0 .
\end{array}\right.
$$

Taking into account estimate (17) given by Lemma 3.1, then by using standard elliptic regularity (see e.g. [14]) for the boundary value problem (24) we find that

$$
\left\|\frac{\partial}{\partial n}\left(\hat{u}_{\alpha}-\hat{\tilde{u}}_{\alpha}\right)\right\|_{L^{2}(\Gamma)}=O\left(\alpha^{d+1}\right) .
$$

The fact that $\Lambda_{\alpha}(\tilde{f})-\tilde{\Lambda}_{\alpha}\left(\left.\tilde{u}_{\alpha}\right|_{\Gamma \times(0, T)}\right):=\frac{\partial}{\partial n}\left(u_{\alpha}-\tilde{u}_{\alpha}\right) \in L^{\infty}\left(0, T ; L^{2}(\Gamma)\right)$, we deduce, as done in the proof of Lemma 3.1, that

$$
\| \Lambda_{\alpha}(\tilde{f})-\left.\tilde{\Lambda}_{\alpha}\left(\left.\tilde{u}_{\alpha}\right|_{\Gamma \times(0, T)}\right)\right|_{L^{2}(\Gamma)}=O\left(\alpha^{d+1}\right),
$$

which implies that

$\int_{0}^{T} \int_{\Gamma}\left[\theta_{\eta}\left(\Lambda_{\alpha}(\tilde{f})-\tilde{\Lambda}_{\alpha}\left(\left.\tilde{u}_{\alpha}\right|_{\Gamma \times(0, T)}\right)\right)+\partial_{t} \theta_{\eta} \partial_{t}\left(\Lambda_{\alpha}(\tilde{f})-\tilde{\Lambda}_{\alpha}\left(\left.\tilde{u}_{\alpha}\right|_{\Gamma \times(0, T)}\right)\right)\right] d \sigma(x) d t=O\left(\alpha^{d+1}\right)$.

This completes the proof of our Theorem.

The smoothness of $\Omega$ and $\Omega^{\prime}$ used in Theorem 3.1 can be weakened as follows.

REMARK 3.1. After an attentive treatment of our analysis and different approximations, and by referring again to [21] for the regularity of the solutions of (7), we assume that $C^{m}$ - regularity $(m \geq 2)$ would be sufficient for the domains $\Omega$ and $\Omega^{\prime}$.

We are now in position to describe our identification procedure which is based on Theorem 3.1. Let us neglect the asymptotically small remainder in the asymptotic formula (22). Then, it follows

$c_{\alpha}(x)-c_{0}(x) \approx \frac{2}{\alpha^{d-1}} \int_{\mathbb{R}^{d}} \frac{e^{-2 i \eta \cdot x}}{|\eta|^{2}} \int_{0}^{T} \int_{\Gamma}\left(\theta_{\eta}+\partial_{t} \theta_{\eta} \partial_{t} \cdot\right)\left(\Lambda_{\alpha}-\Lambda_{0}\right)(\tilde{f})(x, t) d \sigma(y) d t d \eta, x \in \Omega$.

The method of reconstruction we propose here consists in sampling values of

$$
\frac{1}{|\eta|^{2}} \int_{0}^{T} \int_{\Gamma}\left(\theta_{\eta}+\partial_{t} \theta_{\eta} \partial_{t} \cdot\right)\left(\Lambda_{\alpha}-\Lambda_{0}\right)(\tilde{f})(x, t) d \sigma(x) d t
$$

at some discrete set of points $\eta$ and then calculating the corresponding inverse Fourier transform. 
In the following, it is not hard to prove the more convenient approximation in terms of the values of local Dirichlet to Neumann maps $\Lambda_{\alpha}$ and $\Lambda_{0}$ at $\tilde{f}$.

Corollary 3.1. Let $\eta \in \mathbb{R}^{d}$ and let $\tilde{f}$ be defined by (8). Suppose that $\Gamma$ and $T$ geometrically control $\Omega$, then we have the following approximation

$$
\begin{aligned}
c_{\alpha}(x) \approx & c_{0}(x)-\frac{2}{\alpha^{d-1}} \int_{\mathbb{R}^{d}} \frac{e^{-2 i \eta \cdot x}}{|\eta|^{2}} \int_{0}^{T} \int_{\Gamma}\left[e^{i|\eta| t} \partial_{t}\left(e^{-i|\eta| t} g_{\eta}(y, t)\right)\left(\Lambda_{\alpha}-\Lambda_{0}\right)(\tilde{f})(y, t)\right] \\
(25) \quad & \times d \sigma(y) d t d \eta, x \in \Omega,
\end{aligned}
$$

where the boundary control $g_{\eta}$ is defined by (9).

Proof. The term $\int_{0}^{T} \int_{\Gamma} \partial_{t} \theta_{\eta} \partial_{t}\left(\Lambda_{\alpha}-\Lambda_{0}\right)(\tilde{f})(x, t) d \sigma(x) d t$, given in Theorem 3.1, has to be interpreted as follows:

$\int_{0}^{T} \int_{\Gamma} \partial_{t} \theta_{\eta} \cdot \partial_{t}\left(\Lambda_{\alpha}-\Lambda_{0}\right)(\tilde{f})(x, t) d \sigma(x) d t=-\int_{0}^{T} \int_{\Gamma} \partial_{t}^{2} \theta_{\eta} \cdot\left(\Lambda_{\alpha}-\Lambda_{0}\right)(\tilde{f})(x, t) d \sigma(x) d t$, because $\left.\theta_{\eta}\right|_{t=T}=0$ and $\left.\partial_{t}\left(\frac{\partial u_{\alpha}}{\partial n}-\frac{\partial u}{\partial n}\right)\right|_{t=0}=0$. In fact, in view of the ODE (11), the term $\int_{0}^{T} \int_{\Gamma}\left[\theta_{\eta}\left(\Lambda_{\alpha}-\Lambda_{0}\right)+\partial_{t} \theta_{\eta} \cdot \partial_{t}\left(\Lambda_{\alpha}-\Lambda_{0}\right)\right] \tilde{f}(x, t) d \sigma(x) d t$ may be simplified after integration by parts over $(0, T)$ and use of the fact that $\theta_{\eta}$ is the solution to the ODE (11) to become

$$
-\int_{0}^{T} \int_{\Gamma} e^{i|\eta| t} \partial_{t}\left(e^{-i|\eta| t} g_{\eta}\right) \cdot\left(\Lambda_{\alpha}-\Lambda_{0}\right)(\tilde{f})(x, t) d \sigma(x) d t .
$$

Then, the desired approximation is established.

4. Conclusion. The use of approximate formula (22), including the difference between the local Dirichlet to Neumann maps, represents a promising approach to the dynamical identification and reconstruction of a coefficient which is unknown in a bounded domain (but it is known outside) for a class of hyperbolic PDE. We believe that this method will yield a suitable approximation to the dynamical identification of small conductivity ball (of the form $z+\alpha D$ ) in a homogeneous medium in $\mathbb{R}^{d}$ from the boundary measurements. We will present convenable numerical implementations for this investigation. This issue will be considered in a forthcoming work.

\section{REFERENCES}

[1] N. U. Ahmed, And T. WAn, Exact boundary controllability of electromagnetic fields in general regions, Dynam. Systems Appl., 5 (1996), pp. 229-243.

[2] H. AmmaRI, Identification of small amplitude perturbations in the electromagnetic parameters from partial dynamic boundary measurements, J. Math. Anal. Appl., 282 (2003), pp. 479494.

[3] C. Bardos, G. Lebeau, And J. Rauch, Sharp sufficient conditions for the observation, control and stabilization of waves from the boundary, SIAM J. Control Opt., 30 (1992), pp. 10241065.

[4] L. Beilina And M. V. KliBanov, A globally convergent numerical method for a coefficient inverse problem, SIAM J. Sci. Comp., 31:1 (2008), pp. 478-509.

[5] L. Beilina AND M. V. Klibanov, A globally convergent numerical method and the adaptivity technique for a hyperbolic coefficient inverse problem. Part I: analytical study, available on-line (2009). 
[6] M. I. BeLishev, Dynamical inverse problem for the equation $u_{t t}-\Delta u-\nabla \ln \rho \cdot \nabla u=0$ (the $B C$ method), Cubo 10, 2 (2008), pp. 15-30.

[7] M. I. Belishev, The Caldéron problem for two-dimensional manifolds by the BC-method, SIAM J. Math. Anal., 35:1 (2003), pp. 172-182.

[8] M. I. Belishev And YA Kurylev, Boundary control, wave field continuation and inverse problems for the wave equation, Comput. Math. Appl., 22 (1991), pp. 27-52.

[9] H. BrezIs, Analyse fonctionnelle, Masson, Paris, 1983.

[10] G. Bruckner And M. Yamamoto, Determination of point wave sources by pointwise observations: stability and reconstruction, Inverse Problems, 16 (2000), pp. 723-748.

[11] D. G. Cedio-Fengya, S. Moskow And M. Vogelius, Identification of conductivity imperfections of small diameter by boundary measurements. Continuous dependence and computional reconstruction, Inverse Problems, 14 (1998), pp. 553-595.

[12] Y. Chen, Inverse scattering via heisenberg uncertainty principle, Inverse Problems, 13 (1997), pp. 253-282.

[13] C. Daveau, A. Khelifi, and A. Sushchenko, Reconstruction of closely spaced small inhomogeneities via boundary measurements for the full time-dependent Maxwell's equations, Appl. Math. Modelling, 33:3 (2009), pp. 1719-1728.

[14] L. C. Evans, Partial Differential Equations. Graduate Studies in Mathematics, AMS, Providence, Rhode Island 1998.

[15] A. Friedman And M. Vogelius, Identification of small inhomogeneities of extreme conductivity by boundary measurements: a theorem on continuous dependence, Arch. Rat. Mech. Anal., 105 (1989), pp. 299-326.

[16] S. I. KabanikHin, Projection-difference methods for determining the coefficients of hyperbolic equations, Nauka, Novosibirsk, 1988.

[17] M. V. Klibanov, Inverse problems and carleman estimates, Inverse Problems, 8 (1991), pp. $575-596$.

[18] M. Klibanov and A. A. Timonov, Numerical studies on the globally convergent convexification algorithm in 2d, Inverse Problems, 23 (2007), pp. 123-138.

[19] M. V. Klibanov and A. Timonov, Carleman Estimates for Coefficient Inverse Problems and Numerical Applications, VSP, Utrecht, The Netherlands, 2004.

[20] J. Xin AND M. V. Klibanov, Comparative studies of the globally convergent convexification al- gorithm with application to imaging of antipersonnel land mines, Applicable Analysis, 86 (2007), pp. 1147-1176.

[21] J. L. Lions, Contrôlabilité exacte, Perturbations et Stabilisation de Systèmes Distribués, Tome 1, Contrôlabilité Exacte, Masson 1988, Paris.

[22] A. Nachman, Global uniqueness for a two-dimensional inverse boundary value problem, Ann. Math., 143 (1996), pp. 71-96.

[23] L. PäIVäRINTA AND V. Serov, Recovery of jumps and singularities in the multidimensional Schrödinger operator from limited data, Inverse Probl. Imaging, 1:3 (2007), pp. 525-535.

[24] J. P. Puel and M. Yамамото, Applications de la contrôlabilité exacte à quelques problemes inverses hyperboliques, C. R. Acad. Sci. Paris, Série I, 320 (1995), pp. 1171-1176.

[25] J. P. Puel And M. YAmamoto, On a global estimate in a linear inverse hyperbolic problem, Inverse Problems, 12 (1996), pp. 995-1002.

[26] Rakesh AND W. Symes, Uniqueness for an inverse problem for the wave equation, Comm. Part. Differ. Equat., 13 (1988), pp. 87-96.

[27] A. G. Ramm, Inverse Problems: Mathematical and Analytical Techniques with Applications to Engineering, Springer US 2005.

[28] A. G. Ramm And Rakesh, Property $C$ and an inverse problem for a hyperbolic equation, J. Math. Anal. Appl., 156 (1991), pp. 209-219.

[29] V. SERov AND L. PäIVäRINTA, Inverse scattering problem for two-dimensional Schrödinger operator, J. Inv. Ill-Posed Problems, 14:3 (2006), pp. 295-305.

[30] J. Sylvester, And G. Uhlmann, A global uniqueness theorem for an inverse boundary value problem, Ann. Math., 125 (1987), pp. 153-169.

[31] Z. Sun, On the continuous dependence for an inverse initial boundary value problem for the wave equation, J. Math. Anal. Appl., 150 (1990), pp. 188-204.

[32] М. Yамамото, On an inverse problem of determining source terms in Maxwell's equations with a single measurement, Inverse problems, tomography, and image processing (Plenum, New York), 15 (1998), pp. 241-256.

[33] М. Yамамото, Stability, reconstruction formula and regularization for an inverse source hyperbolic problem by a control method, Inverse Problems, 11 (1995), pp. 481-496. 